\title{
LA PERSONALIDAD DEL TURISTA COMO CRITERIO DE SEGMENTACIÓN DE DESTINOS DE SOL Y PLAYA: UNA APLICACIÓN AL DESTINO GRAN CANARIA
}

\author{
Noelia Medina-Ojeda \\ Universidad de Las Palmas de Gran Canaria \\ https://orcid.org/0000-0001-6406-0473 \\ Josefa D. Martín-Santana \\ Universidad de Las Palmas de Gran Canaria \\ https://orcid.org/0000-0002-7078-0271 \\ Diego R. Medina-Muñoz \\ Universidad de Las Palmas de Gran Canaria \\ https://orcid.org/0000-0002-0370-2353
}

\section{RESUMEN}

El objetivo de este trabajo es analizar la influencia de las características sociodemográficas en la personalidad del turista en aras de justificar su potencial como criterio de segmentación de mercados turísticos de sol y playa. Para medir la personalidad se utilizó el método BIG FIVE y el rasgo de personalidad denominado búsqueda de sensaciones, aplicados a una muestra de 450 turistas de Gran Canaria (España). Los resultados muestran que las características sociodemográficas influyen en la personalidad de los turistas, siendo idónea, por tanto, como criterio de segmentación de mercados.

Palabras clave: Rasgos de personalidad del turista: los cinco grandes; búsqueda de sensaciones; perfil sociodemográfico.

Tourist personality of the tourist as segmentation criteria for sun and beaches destinations: an application to the destination gran Canaria

Fecha de recepción: 27 de marzo de 2018

Fecha de aceptación: 5 de noviembre de 2018

Facultad de Economía, Empresa y Turismo. Universidad de Las Palmas de Gran canaria Campus Universitario de Tafira. 35017 LAS PALMAS DE GRAN CANARIA(España).E-mail: noelia.medina@ulpgc.es, josefa.martin@ulpgc.es, diego.medina@ulpgc.es 


\begin{abstract}
The purpose of this study is to examine the influence of socio-demographic characteristics on tourist personality in order to be used as segmentation criteria for sun and beaches tourists. The method used to measure personality is the "Big 5" and the personality trait referred to as sensation seeking. A sample of 450 tourists was carried out on Gran Canaria (Spain). According to the results, socio-demographic characteristics influence on tourist personality traits, and therefore they could be of interest as a tourist market segmentation criteria.
\end{abstract}

Keywords: Tourist's personality; big five; sensation seeking; sociodemographic data.

\title{
1. INTRODUCCIÓN
}

El turismo se ha convertido en un sector clave en la economía mundial, y más especialmente en destinos maduros ya consolidados, en los que es preciso apostar por la diferenciación como estrategia para poder mantener cifras positivas cada año, que sólo puede conseguirse a través de una buena segmentación basada en un conocimiento más profundo de los turistas, más allá de sus características sociodemográficas (Laesser y Zehrer, 2012).

Tradicionalmente, la segmentación en turismo se ha realizado en base a criterios sociodemográficos y económicos, sin tener en cuenta la valiosa herramienta que supone el conocimiento de la personalidad de nuestros turistas. El hecho de que la personalidad implique patrones estables y duraderos de pensamiento, emociones y comportamientos presentes en cada individuo, que lo hacen distinto al resto (Carver y Scheier, 2008; Larsen y Buss, 2008), le convierte en un criterio fiable para la segmentación.

En el contexto turístico, la investigación sobre el tópico de la personalidad ha quedado limitada a la elaboración de distintas tipologías (Cohen, 1972; Smith, 1977; Holland, 1985; Plog, 1991) que toman como criterio de clasificación, entre otros, las actividades turísticas que realizan los turistas o el tipo de viaje independiente o paquete turístico comprado. De ahí, que en la actualidad, algunos autores demanden otros criterios a la hora de plantear la personalidad del turista, más allá de esas tipologías (Gretzel et al., 2004). Sin embargo, se trata de un constructo bastante controvertido debido a que, por una parte, no se han establecido criterios universales para identificar perfiles de personalidad y, por otra parte, a la diversidad de enfoques o perspectivas distintas desde los que es abordada (Schneider y Vogt, 2012).

Durante los últimos años, se ha producido un aumento del interés por la personalidad vinculada al contexto turístico, como lo evidencian las investigaciones académicas analizadas en este trabajo entre los años 1999 y 2015. La variedad de rasgos de personalidad y de contextos turísticos que han sido considerados en dichas investigaciones podría explicar, al menos parcialmente, la diversidad de las evidencias disponibles sobre la asociación entre la personalidad de los turistas y el perfil sociodemográfico (Pizam, 2004; Xu et al., 2012). 
En concreto, los seis rasgos de personalidad que han recibido una mayor atención en la literatura sobre personalidad y turismo son la búsqueda de sensaciones y los cincos grandes rasgos de personalidad (extroversión, agradabilidad, apertura a la experiencia, responsabilidad y neurotismo) que integran el Modelo de los Cinco Grandes, siendo Costa y McCrae, Eysenk y Catt

ell los autores que más los han investigado y evaluado (Paunonen y Jackson, 2000; Mueller y Peters, 2008).

Sobre la base de estas consideraciones, se plantea como objetivo principal de este trabajo establecer un modelo de medida de la personalidad integrado por los cinco grandes rasgos de personalidad, conocidos como los Cinco Grandes, y el rasgo de personalidad denominado búsqueda de sensaciones. Adicionalmente, se plantea como segundo objetivo analizar la influencia de las características sociodemográficas en la personalidad de los turistas al objeto de conocer la idoneidad de esta variable como criterio de segmentación de mercados en el sector turístico al poder establecer patrones duraderos y fiables.

\section{REVISIÓN DE LA LITERATURA}

¿Por qué cada persona elige un destino turístico y unos productos turísticos determinados? ¿Qué determina su elección, además de los conocidos factores "pullpush"? (Crompton, 1979; Dann, 1977). Para algunos autores, como McCrae y Costa (2003) la respuesta a estas preguntas puede encontrarse en la personalidad. A pesar de su importancia, se trata de un constructo bastante controvertido debido a la inexistencia de criterios universales para identificar perfiles de personalidad, así como a la diversidad de enfoques o perspectivas desde los que es abordada.

La personalidad es la organización dinámica dentro del individuo de aquellos sistemas psicofísicos que determinan sus ajustes únicos al ambiente, y su desarrollo tiene lugar a través de las siguientes etapas: sentido corporal, autoidentidad, valoración del yo, extensiones del yo, autoimagen, agente racional, lucha propia y el sí mismo (Allport, 1940). Para autores más contemporáneos (Carver y Scheier, 2008; Larsen y Buss, 2008), la personalidad implica los patrones estables y duraderos de pensamiento, emociones y comportamientos presentes en cada individuo, que lo hacen distinto al resto. Los rasgos de personalidad se emplean para representar la tendencia básica de la persona que, en relación con el entorno, produce adaptaciones características, tales como habilidades, creencias y actitudes (Feldt, Metsäpelto, Kinnunen y Pulkkinen, 2007).

Tal y como comentábamos en los párrafos anteriores, se observa una gran diversidad de enfoques o perspectivas desde los que es abordada la personalidad, y dentro de ellas la más aceptada es la perspectiva de los rasgos cuyo objetivo es analizar la estructura de la personalidad a través de la medida, identificación y clasificación de las similitudes y diferencias de los rasgos de la personalidad. Atendiendo a esta perspectiva, la unidad primaria de la personalidad es el rasgo, puesto que es razonablemente más estable en el tiempo y relativamente consistente y es lo que diferencia a unos individuos de otros (Allport, 1961; Carver y Scheier, 2008; Larsen y Buss, 2008). Los rasgos pueden estudiarse de manera idiográfica (rasgos individuales) o nomotética (rasgos comunes). Para muchos, el enfoque idiográfico describe a los individuos de manera más adecuada. La evidencia de los rasgos 
proviene de muchas fuentes: lenguaje, conducta, documentos (como las cartas) y cuestionarios como el Estudio de los Valores (Cloninger, 2003). Aunque los representantes de la perspectiva de los rasgos más reseñados en los estudios de turismo son Eysenck, Allport, Cattell, y Costa y McCrae, cabe destacar, sin embargo, las aportaciones de Allport en el desarrollo de la psicología académica con temas como la consistencia de la personalidad, la influencia social, el concepto de sí mismo y la interacción de la personalidad con la influencia social en la determinación de la conducta (Cloninger, 2003).

También son de relevante importancia, dentro de la perspectiva de los rasgos, las aportaciones de Cattell (1992) a la teoría de la personalidad a través de su teoría de los factores y el Modelo 16PF. Cattell (1992) definió la personalidad simplemente como lo que permite una predicción de lo que hará una persona en una situación dada. Utilizó como método de investigación principalmente el análisis factorial a partir del cual desarrolló un gran número de test de personalidad. Para su investigación se basó en tres fuentes de datos: cuestionarios de autor reporte, test objetivos -incluidos test proyectivos y medidas conductuales- e información de la historia vital. Su instrumento de medida de personalidad $16 \mathrm{PF}$, con la que mide los 16 principales rasgos origen de la personalidad, se basa en varios tipos de rasgos: dinámicos, de temperamento y habilidad.

En la actualidad, uno de los métodos más utilizados para medir la personalidad, basado en el método factorial utilizado por Cattell (1992), se basa en cinco factores y es el método conocido como los Cinco Grandes (Barrick y Mount, 1991; Goldberg, 1990; McCrae y Costa, 1987), cuyo principal objetivo es describir la personalidad, y no sus causas. Costa y McCrae (1990) sostienen que el Modelo de los Cinco Grandes ha contribuido notablemente a la modelización de la estructura de la personalidad al demostrar cómo cinco dimensiones, de base biológica, pueden abarcar la mayoría de los rasgos de personalidad que se recogen en la literatura. Por tanto, los factores agradabilidad, responsabilidad, extroversión, neurotismo y apertura a la experiencia son los cinco grandes factores dimensionales a partir de los cuales se constituye la personalidad (Ledesma, Sánchez y Díaz-Lázaro, 2011). Estos cinco factores básicos de la personalidad se obtuvieron a partir de los análisis factoriales aplicados a las palabras o a los términos que las personas utilizan en el lenguaje cotidiano para describir la personalidad de un sujeto, es decir, el enfoque léxico de la personalidad (Goldberg, 1981), de ahí la preocupación por los estudios transculturales llevados a cabo en el marco del Modelo de los Cinco Grandes (Benet-Martínez y John, 1998; McCrae et al., 2000), ya que una de las premisas de partida de este modelo es que la personalidad se basa en procesos universales que trascienden las improntas culturales. Es por ello que esta estructura de cinco factores debería replicarse en diferentes culturas. Esta preocupación ha traído consigo que sus dimensiones hayan sido etiquetadas con diferentes nombres a raíz de la utilización reiterada de análisis factoriales aplicados a diferentes test de personalidad y calificaciones de personalidad de observadores externos (Ledesma, Sánchez y DíazLázaro, 2011).

Concretamente, el Modelo de los Cinco Grandes sostiene la existencia de cinco amplias dimensiones de personalidad, divididas cada una de ellas en seis facetas, que abarcan la mayor parte de los rasgos de personalidad que existen. Para McCrae et al. (2000), este modelo ha eliminado la rivalidad existente entre los diferentes sistemas 
de la estructura de la personalidad al demostrar que la mayoría de los rasgos pueden entenderse en términos de cinco dimensiones básicas, independientemente de la cultura o el lenguaje de las personas, y que además estos rasgos se mantienen relativamente estables a lo largo de la vida. Lo que plantean estos autores es que desde el nacimiento cada persona tendría una orientación genética que predispone lo que va a ser o lo que puede llegar a ser su personalidad. Estos cinco factores se consideran como la solución adecuada e intermedia entre modelos extremos, muy amplios o muy escuetos, como son el modelo de tres factores de Eysenk o los interminables dieciséis factores de Cattell (Paunonen y Jackson, 2000; Mueller y Peters, 2008). De ahí, que sea utilizado por muchos investigadores en el contexto turístico para abordar tópicos de investigación tan variados como el turismo de reuniones corporativas (Ariffin, Ahmad y Ishak, 2008), el turismo de salud (Hee y Johari, 2014), el turismo de ocio turístico (Kalmus, Realo y Siibak, 2011), el turismo vacacional (Kroesen y Handy, 2014; Passafaro et al., 2015), el turismo de snowboarding (Mueller y Peters, 2008) y el turismo de deportes de riesgo (Tok, 2011).

Este Modelo de los Cinco Grandes -que ha sido de gran utilidad en el estudio de la influencia de la personalidad como factor interno que influye en el comportamiento del consumidor bajo la premisa de que los rasgos de personalidad influyen en el comportamiento humano (Hee y Johari, 2014)- se ha aplicado en investigaciones muy diversas que han tenido como punto de convergencia el análisis de la influencia de la los cinco grandes rasgos de la personalidad en las creencias, actitudes y conductas de los individuos. Así, Kroesen y Handy (2014) analizaron la influencia de la personalidad en el comportamiento de ocio y la práctica deportiva porque, según estos autores, estas cinco dimensiones son factores idóneos para predecir el tipo de actividades de ocio y deportivas que se realizan, ya que permanecen constantes a lo largo de la vida. Por su parte, Kalmus, Realo y Siibak (2011) estudiaron la influencia de la personalidad, utilizando este modelo de los cinco rasgos en la búsqueda de información turística y el uso de Internet. Es más, este modelo se ha utilizado en múltiples ocasiones para determinar la influencia de la personalidad en la elección del tipo de viaje o de un destino determinado (Abbate y Di Nuovo, 2013; Ariffin, Ahmad y Ishak, 2008), así como en aquellos trabajos en los que se pretende conocer qué factores de la personalidad influyen en la elección de un destino o tipo de viaje determinado en función de las distintas motivaciones, ya sean inherentes al individuo o asociadas a los atributos del destino turístico en sí.

Existe un cierto consenso en la literatura, como se pone de manifiesto en el trabajo de Cloninger (2003), relativo a su idoneidad como instrumento de medición por la consistencia transituacional de los rasgos, su base genética, su estabilidad temporal y su estructura universal. Precisamente, en relación a la estructura del modelo y especialmente en los últimos años, ha surgido entre algunos autores el debate en torno al número de sus dimensiones (Paunonen y Jackson, 2000), ya que en la literatura hay evidencia empírica de que el modelo se compone de tres (Eysenck, 1991), cinco (McCrae y Costa, 1987), seis (Jackson, Paunonen, Fraboni, y Goffin, 1996), e incluso 16 (Cattell y Krug, 1986). Sin embargo, la mayoría de los investigadores coinciden en que el modelo de personalidad conocido como los Cinco Grandes está integrado por la extroversión, la agradabilidad, la responsabilidad, el neurotismo, y la apertura a la experiencia (Feldt et al., 2007). 
De acuerdo con Costa y McCrae (1992), Mueller y Peters (2008) y Kalmus, Realo y Siibak (2011) estas cinco dimensiones (con su polo opuesto entre paréntesis) se pueden definir conforme a los párrafos que se exponen a continuación.

La dimensión extroversión (introversión) evalúa la sociabilidad, la facilidad para comunicarse con los demás, la asertividad y la facilidad para iniciar y mantener conversaciones. Este rasgo se asocia con emociones positivas y con la tendencia a estar activo, buscar estímulos y disfrutar de la compañía de los demás. Los adjetivos que definen a la persona extrovertida son entusiasta, llena de energía, optimista, amigable, comunicativa y colaboradora. El polo negativo es propio de personas con tendencia al aislamiento o el retraimiento, que podrían ser calificadas como calladas, reservadas, obedientes, impasibles, controladas y sosegadas.

La dimensión agradabilidad (oposicionismo) evalúa la capacidad para establecer vínculos psicosociales y la disposición a preocuparse por los demás. Los individuos con este rasgo de personalidad podrían ser calificados como agradables, fiables, afectuosos, colaboradores y conciliadores. En casos extremos, el polo negativo se asocia a la psicopatía, y los adjetivos para calificar a este tipo de persona son fría, poco amistosa, rencorosa, cínica, poco colaboradora, irritable y manipuladora.

La dimensión responsabilidad (negligencia) evalúa la capacidad para actuar de acuerdo a propósitos o metas claras para poder organizar y llevar adelante proyectos e ideas. Las personas con predominio de este rasgo pueden ser calificadas como prudentes, fiables, organizadas, puntuales, diligentes, metódicas y ambiciosas. El polo negativo es propio de personas que andan sin rumbo u objetivo, o resultan indolentes y que podrían ser descritas como imprudentes, poco cuidadosas, impuntuales, poco fiables, perezosas y sin carácter.

La dimensión neurotismo (estabilidad emocional) incluye aspectos ligados al bienestar o malestar psicológico, al afecto y a las emociones negativas. Evalúa la inestabilidad emocional, la tendencia a experimentar emociones negativas como miedo, sentimiento de culpa, tristeza o enojo. Las personas en las que predomina esta dimensión pueden ser denominadas como nerviosas, inestables, ansiosas, impulsivas e hipocondríacas. El polo negativo lo constituyen personas sosegadas, emocionalmente estables, seguras de sí mismas y vanidosas.

Y, para concluir, la dimensión apertura a la experiencia (convencionalismo) evalúa la presencia de una imaginación activa, sensibilidad estética, capacidad de introspección y curiosidad intelectual. Los adjetivos aplicables al individuo con predominio de este rasgo son ingenioso, imaginativo, inventivo, curioso y poco convencional. En el polo negativo encontramos a individuos que son superficiales, convencionales, poco originales, poco creativos y sencillos.

Otra de las características de los Cinco Grandes es la importancia dada a la medición de los constructos teóricos (Costa y McCrae, 1989). Es por esto que se han diseñado varios instrumentos para evaluar la personalidad de acuerdo a las líneas del modelo. El más conocido de estos instrumentos es el Inventario de Personalidad NEO-PI-R de Costa y McCrae (1989), al que siguen el Inventario NEO-FFI (NEO Five-Factory Inventory) (1992) y el Inventario TDA (Trait Descriptive Adjectives) de Goldberg (1990), este último de adjetivos descriptivos de rasgos. Los dos primeros instrumentos (NEO-PI-R y NEO FFI) se estructuran en seis facetas por factor o dimensión (véase Tabla 1). 


\section{Tabla 1}

FACETAS DE LOS PRINCIPALES INSTRUMENTOS PARA LA MEDICIÓN DE LOS CINCO GRANDES

\begin{tabular}{|c|c|c|}
\hline Facetas del neurotismo & NEO-PI-R & NEO FFI \\
\hline Ansiedad & $\mathrm{X}$ & $\mathrm{X}$ \\
\hline Hostilidad & $\mathrm{X}$ & $\mathrm{X}$ \\
\hline Depresión & $\mathrm{X}$ & $\mathrm{X}$ \\
\hline Ansiedad social & $\mathrm{X}$ & $\mathrm{X}$ \\
\hline Impulsividad & $\mathrm{X}$ & $\mathrm{X}$ \\
\hline Vulnerabilidad & $\mathrm{X}$ & $\mathrm{X}$ \\
\hline Facetas de la extroversión & NEO-PI-R & NEO FFI \\
\hline Cordialidad & $\mathrm{X}$ & $\mathrm{X}$ \\
\hline Gregarismo & $\mathrm{X}$ & $\mathrm{X}$ \\
\hline Asertividad & $\mathrm{X}$ & $\mathrm{X}$ \\
\hline Actividad & $\mathrm{X}$ & $\mathrm{X}$ \\
\hline Búsqueda de emociones & $\mathrm{X}$ & $X$ \\
\hline Emociones positivas & $\mathrm{X}$ & $\mathrm{X}$ \\
\hline Facetas de la apertura a la experiencia & NEO-PI-R & NEO FFI \\
\hline Fantasía & $\mathrm{X}$ & $\mathrm{X}$ \\
\hline Estética & $\mathrm{X}$ & $\mathrm{X}$ \\
\hline Sentimientos & $\mathrm{X}$ & $\mathrm{X}$ \\
\hline Acciones & $\mathrm{X}$ & $\mathrm{X}$ \\
\hline Ideas & $\mathrm{X}$ & $\mathrm{X}$ \\
\hline Valores & $\mathrm{X}$ & $\mathrm{X}$ \\
\hline Facetas de la agradabilidad & NEO-PI-R & NEO FFI \\
\hline Confianza & $\mathrm{X}$ & $\mathrm{X}$ \\
\hline Franqueza & $\mathrm{X}$ & $\mathrm{X}$ \\
\hline Altruismo & $\mathrm{X}$ & $\mathrm{X}$ \\
\hline Actitud conciliadora & $\mathrm{X}$ & $\mathrm{X}$ \\
\hline Modestia & $\mathrm{X}$ & - \\
\hline Sensibilidad a los demás & $\mathrm{X}$ & - \\
\hline
\end{tabular}




\begin{tabular}{lcc}
\hline Facetas de la responsabilidad & NEO-PI-R & NEO FFI \\
\hline Competencia & $\mathrm{X}$ & - \\
Orden & $\mathrm{X}$ & - \\
Sentido del deber & $\mathrm{X}$ & - \\
Necesidad de logro & $\mathrm{X}$ & - \\
Autodisciplina & $\mathrm{X}$ & - \\
Deliberación & $\mathrm{X}$ & - \\
\hline
\end{tabular}

Fuente: Elaboración propia a partir de Costa y McCrae (1989 y 1992) y Goldberg (1990).

A pesar de que los Cinco Grandes contienen los principales rasgos de personalidad, otras investigaciones han incluido otros rasgos de personalidad, entre ellos, los que parecen derivarse del temperamento como, por ejemplo, la búsqueda de sensaciones, cuya importancia en el sector turístico nos ha llevado a abordarla conjuntamente con los Cinco Grandes en este estudio.

El temperamento tiene una fuerte componente biológica, de ahí que sea considerado como una predisposición heredada del individuo a mostrar patrones característicos de conducta tales como la emocionalidad, la actividad y la sociabilidad. El temperamento de una persona es observable desde muy temprano y es estable a lo largo de la vida. Los estudios genéticos sostienen que el temperamento es altamente hereditario (Cloninger, 2003).

Dentro de estos rasgos de personalidad derivados del temperamento está el rasgo búsqueda de sensaciones, concepto desarrollado por Zuckerman y otros (Zuckerman, 1979, 1984, 1990; Zuckerman, Eysenck y Eysenck, 1978). La búsqueda de sensaciones es "la búsqueda de sensaciones y experiencias variadas, novedosas, complejas e intensas y la disposición a correr riesgos físicos, sociales, legales y financieros por tener dichas experiencias" (Zuckerman, 1994:13). Este rasgo, al ser un rasgo de temperamento, tiene una alta influencia de la genética. El hecho de que alguien prefiera practicar deportes peligrosos o de riesgo como el paracaidismo o el esquí, o bien que su cupo de emoción esté cubierto con la excitación de un videojuego, refleja su rasgo de búsqueda de sensaciones. En definitiva, el buscador de sensaciones elegirá estímulos sensoriales y sociales (arte, música, actividades físicas, vestidos, amigos, drogas, etc.) capaces de producir sensaciones inusuales. La búsqueda de sensaciones, pues, está asociada a una personalidad con alto nivel de asunción de riesgo. Cuando se habla de riesgos se está haciendo referencia a la probabilidad valorada de un resultado negativo. La persona con niveles altos de búsqueda de sensaciones se caracteriza por su tendencia a hacer aquellas cosas que una persona con nivel bajo en búsqueda de sensaciones consideraría peligrosas y arriesgadas. La diferencia entre unos y otros es la distinta valoración que se hace del riesgo.

Además de mostrar también mucha correlación con otros constructos psicológicos relevantes, especialmente con la impulsividad y el psicoticismo, se ha encontrado que 
las variables sociodemográficas están relacionadas con este rasgo. En Estados Unidos, por ejemplo, los hombres, en comparación con las mujeres, obtienen puntuaciones más elevadas en búsqueda de sensaciones, y también en las facetas búsqueda de experiencia y aventuras, y desinhibición. En Australia, Canadá y España, los hombres, también comparados con las mujeres, puntúan más alto en búsqueda de sensaciones, en búsqueda de experiencia y aventuras, y en susceptibilidad al aburrimiento (Zuckerman, 1994). La búsqueda de sensaciones está, asimismo, relacionada con la edad. Este rasgo de personalidad parece aumentar entre la niñez y la adolescencia -etapa esta última en la que alcanza sus mayores niveles- y se reduce conforme aumenta la edad (Zuckerman, 1994).

En el contexto turístico, otros autores, tomando como referencia los trabajos de Zuckerman, han analizado la relación existente entre la búsqueda de sensaciones y las características sociodemográficas de los turistas. En concreto, Pizam et al. (2004) encontraron diferencias significativas en la probabilidad de tener valores altos y bajos en un rasgo personalidad, que combina la asunción de riesgo y la búsqueda de sensaciones, en función del género, obteniéndose como resultado que los hombres, en comparación con las mujeres, presentan unos niveles más altos en este rasgo de personalidad. También Xu et al. (2012), quienes utilizaron además de la búsqueda de sensaciones otros rasgos relacionados con este rasgo (búsqueda de experiencias, búsqueda de emociones y aventura, y susceptibilidad al aburrimiento), analizaron su relación con las características sociodemográficas y demostraron que la búsqueda de experiencias muestra una moderada correlación negativa entre la edad y dos de los rasgos analizados: la búsqueda de emociones y aventura, y la susceptibilidad al aburrimiento.

Sin embargo, y aunque la personalidad, en el contexto turístico y viajero, se ha centrado principalmente en definir distintas tipologías de turistas en función de las actividades turísticas que realizan (Gretzel et al., 2004), algunos autores han llevado a cabo en los últimos años, especialmente a partir de 2009, investigaciones que tienen como objetivo analizar la relación entre la personalidad y el perfil sociodemográfico del turista, detectándose la existencia de diferencias en los rasgos de la personalidad en función del género, la edad, la nacionalidad, la educación, la profesión, el nivel de ingresos, el estado civil o el número de hijos (Abbate y Di Nuovo, 2013; Boksberger et al., 2011; Chandler y Costello, 2002; Laesser y Zehrer, 2012; Pizam et al., 2004; Tok, 2011; Xu et al., 2012).

En la Tabla 2 se muestran los artículos académicos seleccionados para la revisión de la literatura sobre personalidad y turismo que han analizado la asociación entre los rasgos de personalidad y el perfil sociodemográfico de un individuo. Como puede observarse en dicha tabla, son varias las investigaciones del ámbito turístico que han analizado teórica y/o empíricamente las posibles relaciones entre la personalidad y ciertas características sociodemográficas de un individuo: el género, la edad, la nacionalidad, la educación, la profesión, el nivel de ingresos, el estado civil o el número de hijos. Ahora bien, es preciso señalar que la utilización de medidas de la personalidad muy variadas dificulta cualquier intento de generalización. 


\section{Tabla 2 \\ CARACTERÍSTICAS SOCIODEMOGRÁFICAS ASOCIADAS A LA PERSONA- LIDAD EN LA INVESTIGACIÓN SOBRE PERSONALIDAD Y TURISMO}

\begin{tabular}{ll}
\hline \multicolumn{1}{c}{$\begin{array}{c}\text { Características } \\
\text { sociodemográficas }\end{array}$} & \multicolumn{1}{c}{ Fuentes } \\
\hline Género & $\begin{array}{l}\text { Abbate y Di Nuovo (2013); Boksberger } \text { et al. (2011); Chandler y } \\
\text { Costello (2002); Laesser y Zehrer (2012); Pizam } \text { et al. (2004); Tok } \\
(2011)\end{array}$ \\
\hline Edad & $\begin{array}{l}\text { Boksberger } \text { et al. (2011); Chandler y Costello (2002); Laesser y Zehrer } \\
(2012) ; \text { Pizam } \text { et al. (2004); Xu } \text { et al. (2012) }\end{array}$ \\
\hline Nacionalidad & Pizam et al. (2004) \\
\hline Nivel educativo & $\begin{array}{l}\text { Boksberger } \text { et al. (2011); Chandler y Costello (2002); Laesser y Zehrer } \\
(2012) ; \text { Xu } \text { et al. (2012) }\end{array}$ \\
\hline Profesión & $\begin{array}{l}\text { Boksberger } \text { et al. (2011); Chandler y Costello (2002); Laesser y Zehrer } \\
\text { (2012); Pizam } \text { et al. (2004) }\end{array}$ \\
\hline Nivel de ingresos & Pizam et al. (2004); Xu et al. (2012) \\
\hline $\begin{array}{l}\text { Estado civil y número } \\
\text { de hijos }\end{array}$ & Chandler y Costello (2002); Pizam et al. (2004) \\
\hline $\begin{array}{l}\text { Fuente: Elaboración propia a partir de Abbate y Di Nuovo (2013); Boksberger } \text { } \text { (2t al. (2011); Chandler y Costello } \\
\text { (2002); Laesser y Zehrer (2012); Pizam } \text { et al. (2004); Tok (2011) y Xu } \text { et al. (2012). }\end{array}$
\end{tabular}

Aunque Chandler y Costello (2002) no encontraron diferencias significativas en las categorías del modelo de preferencias de estilo de vida y nivel de actividad de Plog (1995) -i.e., aventureros activos, céntricos activos, dependientes activos, aventureros relajados y dependientes relajados- atendiendo a las características sociodemográficas de 412 visitantes de lugares históricos en el este de Tennessee -género, edad, estado civil, tenencia de hijos, nivel educativo y profesión-, las demás investigaciones analizadas generaron evidencias respecto a la existencia de asociaciones significativas entre algunos rasgos de la personalidad y características sociodemográficas.

De manera específica, en un estudio realizado entre 1.429 jóvenes estudiantes de turismo y dirección de empresas turísticas en universidades de 11 países diferentes, Pizam et al. (2004) identificaron diferencias significativas en la probabilidad de tener valores altos y bajos en un rasgo personalidad que combina la asunción de riesgo y la búsqueda de sensaciones, únicamente en función del género y la nacionalidad, mientras que las restantes variables sociodemográficas no resultaron significativas: edad, nivel educativo, número de hijos y nivel de ingresos. Los resultados evidencian la existencia de significativamente más hombres, en comparación con mujeres, con niveles altos en este rasgo de personalidad, así como una mayor presencia relativa de individuos con niveles elevados en este rasgo de personalidad en ciertos países: Israel, Irlanda y Estados Unidos, en comparación con Gabón, Sudáfrica, Eslovaquia e Italia.

Por su parte, Xu et al. (2012), en su análisis de varios rasgos también relacionados con la búsqueda de sensaciones (de experiencias, de emociones y aventura, y susceptibi- 
lidad al aburrimiento), obtuvieron los siguientes resultados del análisis de la información facilitada por una muestra de 50 participantes en tours organizados para la observación de tormentas en Estados Unidos y Canadá. En primer lugar, no se encontraron diferencias significativas en los niveles de estos rasgos de personalidad en función del nivel de ingresos. En segundo lugar, la búsqueda de experiencias no mostró asociación significativa con ninguna de las condiciones sociodemográficas analizadas. Y, en tercer lugar, se observó una moderada correlación negativa entre la edad y dos de los rasgos analizados - la búsqueda de emociones y aventura, y la susceptibilidad al aburrimiento-, así como una fuerte correlación negativa entre la educación y la búsqueda de emociones y aventura.

En cambio, Zuckerman (1983) y Zuckerman et al. (1978) plantean que, en términos generales, la búsqueda de sensaciones podría variar en función de la cultura y es mayor entre los hombres, aumenta entre los 9 y 14 años, alcanza su mayor nivel antes de los 30 años y a partir de ahí se reduce de manera progresiva. En este sentido, son varios los estudios que han generado evidencias sobre la asociación negativa entre la edad y la búsqueda de sensaciones en contextos vinculados a la práctica de actividades recreativas de riesgo (montañismo, carrera de coches, paracaidismo, ala delta o parapente) (Wagner y Houlihan, 1994; Jack y Ronan, 1998). Con relación al género, las evidencias son contradictorias. Mientras que Jack y Ronan (1998) sugieren que los hombres, en comparación con las mujeres, tienden a mostrar un mayor nivel en búsqueda de sensaciones en actividades de riesgo, otros estudios no revelan una asociación significativa entre este rasgo de personalidad y el género (Llewellyn y Sánchez, 2008; Burnik et al., 2008).

Sin embargo, la edad y la profesión resultaron significativas al explicar el grado de autocongruencia -i.e., la diferencia entre las evaluaciones que un individuo hace de su propia personalidad y la del destino que visita- en un estudio realizado por Boksberger et al. (2011) a partir de las opiniones proporcionadas por 1.898 residentes suizos. Concretamente, entre las personas con más de 55 años, a medida que aumenta la edad se reduce la probabilidad de autocongruencia. Por el contrario, la probabilidad de autocongruencia es mayor entre los individuos con profesiones extremas (e.g., desempleados y altos directivos). Estos autores no encontraron asociaciones significativas entre el grado de autocongruencia y el género y la educación.

En cambio, Laesser y Zehrer (2012) segmentaron la misma muestra suiza en cuatro categorías de personalidad y no observaron diferencias significativas en función de la edad. Por el contrario, mientras que los hombres tendían a pertenecer a los grupos "hacedor" y "materialista", las mujeres lo hacían a los grupos "soñador" y "hedonista". Con relación a la educación y la profesión, estos autores encontraron que los miembros del grupo "hacedor" tienen un elevado nivel educativo y ocupan profesiones de nivel medio-alto, mientras que los pertenecientes a los grupos "hedonista" y "materialista" se caracterizan por niveles medios en el nivel educativo y en la profesión. Finalmente, los individuos denominados "soñadores" muestran niveles variados en estas dos variables sociodemográficas.

En lo que a la existencia de posibles asociaciones entre los cinco grandes rasgos de personalidad y las características sociodemográficas se refiere, la literatura sugiere la exis- 
tencia de diferencias únicamente en función del género. En este sentido, se considera que la personalidad de un individuo se mantiene más o menos estable a lo largo del tiempo, por lo que no debería verse afectada por ciertas condiciones socioculturales que podrían variar con el tiempo, como la edad o el nivel de ingresos (Costa y McCrae, 1988; Jani, 2014).

Los resultados obtenidos por Abbate y Di Nuovo (2013), en un estudio realizado sobre 679 turistas italianos visitantes de un centro religioso en Bosnia-Herzegovina, y por Tok (2011), en una investigación sobre la práctica de actividades deportivas arriesgadas con una muestra de 328 residentes en Turquía, revelan una asociación significativa entre el género y la responsabilidad como rasgo de personalidad. De manera específica, son relativamente más las mujeres, en comparación con los hombres, que alcanzan niveles altos en este rasgo (Abbate y Di Nuovo, 2013) y, además, las mujeres exhiben niveles superiores en este rasgo de personalidad (Tok, 2011). Abbate y Di Nuovo (2013) también destacan que son relativamente más las mujeres con una elevada estabilidad emocional.

También son otros los estudios que sugieren la influencia del género en otros grandes rasgos de la personalidad (Tan y Tang, 2013): agradabilidad, neurotismo, extroversión y apertura a las nuevas ideas. Concretamente, se mantiene que las mujeres podrían mostrar niveles superiores de agradabilidad (e.g., Costa et al., 2001), neurotismo y extroversión (e.g., Burton et al., 2007), y que los hombres tienen una actitud más positiva hacia las nuevas ideas (e.g., Costa et al., 2001). Asimismo, Tan y Tang (2013), apoyándose en investigaciones empíricas acometidas en ámbitos no turísticos, también sugieren la posibilidad de que el género modere la influencia de los cinco grandes rasgos de personalidad sobre el comportamiento del turista.

La variedad de rasgos de personalidad y de contextos turísticos que han sido considerados en las investigaciones analizadas en este epígrafe, podría explicar, al menos parcialmente, la diversidad de las evidencias disponibles sobre la asociación entre el perfil sociodemográfico y la personalidad de los turistas, así como las contradicciones observadas. Por tal motivo, este trabajo pretende evaluar las posibles asociaciones entre las características sociodemográficas de los visitantes de Gran Canaria, cuya oferta turística gira en torno al turismo de sol y playa, y los seis rasgos de personalidad que han recibido una mayor atención en la literatura sobre personalidad y turismo: la búsqueda de sensaciones y los cincos grandes rasgos de personalidad (i.e., extroversión, agradabilidad, apertura a la experiencia, responsabilidad y neurotismo).

Considerando todo lo anterior, en este trabajo se plantea la influencia de las características sociodemográficas en los rasgos de personalidad. De manera específica, se formula una hipótesis para cada una de las siguientes características sociodemográficas: género, edad, nivel de estudios, clase social y nacionalidad. La contrastación de estas hipótesis permitirá concluir si la personalidad del turista puede considerarse como un criterio de segmentación idóneo en el sector turístico.

H1: El género del turista de ocio influye en los rasgos de personalidad.

H2: La edad del turista de ocio influye en los rasgos de personalidad.

H3: El nivel de estudios del turista de ocio influye en los rasgos de personalidad.

H4: La clase social del turista de ocio influye en los rasgos de personalidad.

H5: La nacionalidad del turista de ocio influye en los rasgos de personalidad. 


\section{MÉTODO}

El trabajo empírico se ha fundamentado en una encuesta personal realizada, con la ayuda de encuestadores, a turistas extranjeros o españoles no residentes en las Islas Canarias durante los tres últimos días de su viaje de ocio/placer en Gran Canaria, que se implementó durante los meses de mayo, junio y julio de 2015.

Para determinar el tamaño y las características de la población objeto de estudio a través del muestreo no probabilístico, y así definir el perfil de la muestra teórica, se utilizaron diversas fuentes de información secundaria -Instituto Canario de Estadística (ISTAC), Patronato de Turismo de Gran Canaria y FRONTUR-, a partir de las cuales se determinó, además de su tamaño, la distribución de la población en base a una serie de características sociodemográficas de los turistas recibidos durante los meses de mayo, junio y julio de 2014 en Gran Canaria, que era el período en el que se iba a realizar el trabajo de campo en 2015. Las características que se han utilizado para establecer las cuotas a respetar en la determinación del perfil de la muestra fueron nacionalidad, sexo, edad y tipo de alojamiento (véase Tabla 3). El tamaño muestral definido ascendió a 450 turistas, lo que ha supuesto asumir una tasa de error del $\pm 4,62 \%$ con un coeficiente de fiabilidad del $95,5 \%$ y donde $p$ y $q$ son iguales al 50\%. Asimismo, es de destacar que la distribución de la muestra real, en términos generales, es muy similar a la muestra teórica o definida, por lo que su representatividad de la población objeto de estudio está garantizada (véase Tabla 3).

Con respecto al cuestionario, éste se diseñó teniendo en cuenta los múltiples modelos de clasificación de la personalidad existentes, de entre las cuales se eligió como constructo esencial el modelo teórico más aceptado, estudiado y aplicado en la actualidad para evaluar la personalidad: el Modelo de los Cinco Grandes (Big Five), cuya principal ventaja, con respecto al resto de modelos, consiste en que abarca todas las facetas comunes entre todos los sistemas de rasgos de personalidad (John y Srivastava, 1999). Se diseñó una escala para medir los cinco grandes rasgos de la personalidad a partir de la escala Ten Item Personality Inventory (TIPI) (Gosling, Rentfrow y Swann, 2003) y de la escala Big Five Inventory (BFI) (John y Srivastava, 1999).

La escala resultante recoge tres ítems destinados a medir cada una de las cinco dimensiones de la personalidad de los turistas recogidas en el TIPI: neurotismo, extroversión, apertura a la experiencia, agradabilidad y responsabilidad. Los 15 ítems que conforman esta escala se corresponden con una escala tipo Likert de 7 puntos, en la que 1 significa "totalmente en desacuerdo" y 7 "totalmente de acuerdo".

Po otra parte, se decidió añadir una escala para medir el rasgo de personalidad búsqueda de sensaciones (BS), al objeto de determinar si los turistas encuestados en un destino de sol y playa, como es Gran Canaria, presentan niveles altos o bajos en este rasgo y si existe conexión entre los índices de BS de nuestros turistas con la selección de nuestro destino y productos turísticos. Para ello, se seleccionó la escala Brief Sensation Seeking Scale (BSSS) (Hoyle, 2002), que consta de ocho ítems agrupados en cuatro dimensiones (búsqueda de emociones y aventuras; búsqueda de experiencias; desinhibición; susceptibilidad al aburrimiento). Ahora bien, en aras de evitar reiteraciones y hacer lo más reducido posible el cuestionario, se utilizó un solo ítem por dimensión, escogiendo aquel ítem con mayor carga factorial y que, por lo tanto, mejor representara la esencia de cada dimensión 


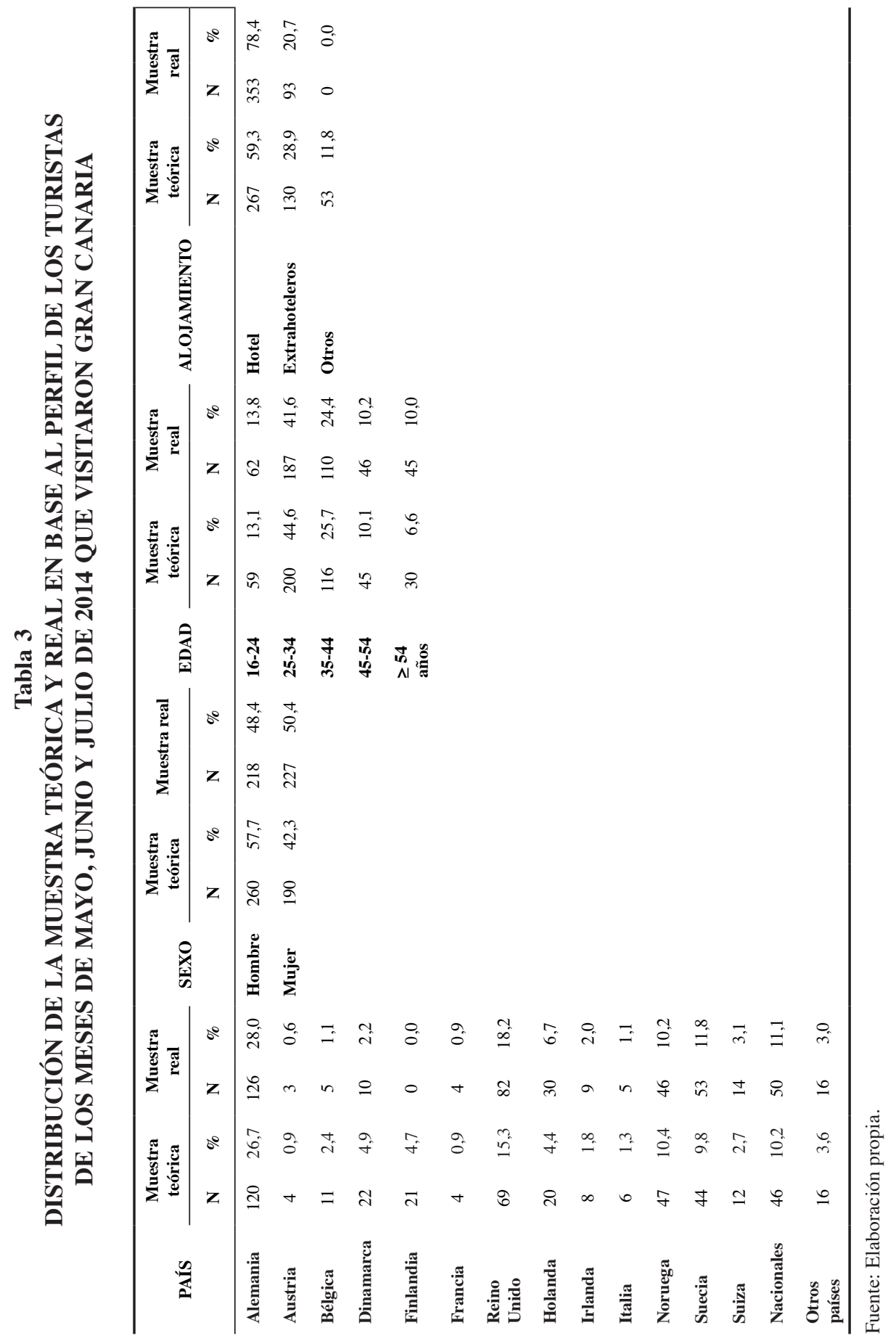


y de ésta sólo se mantuvo uno de los dos ítems recogidos en las cuatro facetas. Como criterio en la elección de los ítems se utilizó la representatividad de los mismos con respecto a la faceta en la que se engloba. En cuanto a la valoración del grado de acuerdo del turista con cada uno de los cuatro ítems, estos son valorados en una escala tipo Likert de 7 puntos, en la que 1 significa "Totalmente en desacuerdo" y 7 "Totalmente de acuerdo".

Finalmente, en el cuestionario también se recogía información de carácter sociodemográfico, es decir, género, edad, nacionalidad, nivel de estudios y profesión del encuestado, así como el nivel de estudios y la profesión de la persona que percibía los mayores ingresos en la unidad familiar para determinar la clase social del encuestado.

Antes de proceder al diseño del cuestionario definitivo en sus tres versiones (española, alemana e inglesa) y al objeto de garantizar la correcta comprensión del mismo, se realizó una primera revisión del mismo entre seis técnicos de los departamentos de Marketing, Producto Turístico y Mercados del Patronato de Turismo de Gran Canaria. Posteriormente, se pretestó con cinco turistas elegidos al azar, que formaban parte de la población objeto de estudio, con el fin de detectar qué ítems presentaban dudas o ambigüedades, especialmente en las versiones alemana e inglesa, que pudieran llevar a una interpretación errónea y a la confusión del encuestado a la hora de valorarlos.

Las encuestas fueron realizadas por cinco encuestadores, todos ellos con titulación universitaria y total dominio de los idiomas alemán e inglés, que además recibieron formación para poder acometer con éxito las entrevistas a los turistas que finalmente participaron en la investigación.

En la Tabla 4 se muestra de manera esquematizada las principales características del proceso metodológico seguido en esta investigación.

Tabla 4

\section{FICHA TÉCNICA DEL PROCESO METODOLÓGICO}

\begin{tabular}{ll}
\hline Procedimiento metodológico & $\begin{array}{l}\text { Encuestas personales administradas por encuestadores en los } \\
\text { idiomas español, inglés y alemán }\end{array}$ \\
\hline Delimitación del universo & $\begin{array}{l}\text { Turistas de ocio/placer de Gran Canaria con } 16 \text { o más años de } \\
\text { edad y no residente en las Islas Canarias }\end{array}$ \\
\hline Ámbito & Gran Canaria \\
\hline Forma de contacto & Personal \\
\hline Muestra definida y real & 450 \\
\hline Error muestral & $\pm 4,62 \%$ \\
\hline Fecha trabajo de campo & Del 25 de mayo al 23 de julio de 2015 \\
\hline
\end{tabular}

Fuente: Elaboración propia

El trabajo de campo abarcó desde el 25 de mayo al 23 de julio de 2015, durante el cual se realizaron 450 encuestas válidas. El análisis del perfil sociodemográfico de la muestra pone de manifiesto que, en lo que al género se refiere, presenta una distribución homogénea entre ambos sexos, ya que un $48,4 \%$ de los encuestados son hombres y el $50,4 \%$ 
restante mujeres. En cuanto a la edad, los tramos con mayor porcentaje se corresponden con los comprendidos entre los 25 y los 44 años, que suponen el 66\% de la muestra total. En relación con el nivel de estudios es de destacar el elevado porcentaje de universitarios de nivel medio y superior $(60,9 \%)$. En cuanto a la clase social, los encuestados pertenecen mayoritariamente a la clase media-media y media-alta $(36,9 \%$ y $32,2 \%$, respectivamente). Alemania (28,0\%), Reino Unido (20,2\%) y Países Nórdicos $(24,2 \%)$ representan el 72,4\% de la muestra, seguidos de España (Península y Baleares) con una representación del $11,1 \%$ y de Holanda con un $6,7 \%$.

\section{RESULTADOS}

\section{Análisis de la dimensionalidad y la validez de la escala de personalidad}

Para determinar la dimensionalidad y validar la escala de personalidad diseñada se ha utilizado un análisis factorial confirmatorios (AFC) utilizando como método de estimación el de máxima verosimilitud. El modelo de medida, como era de esperar, está conformado por seis dimensiones que han sido etiquetadas como "extroversión", "agradabilidad", "responsabilidad", "neurotismo", "apertura a la experiencia" y "búsqueda de sensaciones", que se corresponden con las clásicas dimensiones recogidas en la literatura existente sobre personalidad. No obstante, y para analizar la naturaleza multidimensional de esta escala, se estimó un nuevo modelo en el que todos los ítems se vinculan a un único factor. Los resultados de este nuevo modelo $\left[\chi^{2}(90)=839,56, p=0,000, \mathrm{CFI}=0,52\right.$, RMSEA $=0,14]$ evidencian la idoneidad de un modelo multidimensional $\left[\chi^{2}(75)=210,709\right.$, $p=0,000, \mathrm{CFI}=0,91, \mathrm{RMSEA}=0,06]$, ya que presenta mejores niveles de significación e índices de ajuste. De hecho, el análisis de diferencias de chi-cuadrado arroja la existencia de diferencias significativas (Dif. $\chi^{2}=628,85$, Dif. g.d.l.=15, $p=0,000$ ). Como se muestra en la Tabla 5, el modelo de medida de la personalidad ha quedado formado finalmente por 15 ítems.

\section{Tabla 5}

\section{ÍTEMS DEFINITIVOS DE LA ESCALA DE PERSONALIDAD}

\begin{tabular}{ccl}
\hline DIMENSIONES & CÓDIGO & \multicolumn{1}{c}{ ÍTEM } \\
\hline \multirow{2}{*}{ Extroversión } & V7 & Extrovertida, entusiasta \\
& V8 & Reservada, callada \\
& V9 & Llena de energía \\
\hline \multirow{2}{*}{ Agradabilidad } & V10 & Colaboradora, generosa \\
& V11 & Amable, cálida \\
\hline \multirow{2}{*}{ Responsabilidad } & V13 & Fiable, autodisciplinada \\
& V14 & Desorganizada, poco cuidadosa \\
& V15 & Distraída \\
\hline
\end{tabular}




\begin{tabular}{ccc}
\hline DIMENSIONES & CÓDIGO & \multicolumn{1}{c}{ ÍTEM } \\
\hline Neurotismo & V17 & $\begin{array}{l}\text { Nerviosa, fácil de alterar } \\
\text { Preocupada en exceso }\end{array}$ \\
\hline $\begin{array}{c}\text { Apertura a la } \\
\text { experiencia }\end{array}$ & V19 & Abierta a nuevas experiencias, inventiva \\
V20 & Ingeniosa \\
\hline Búsqueda de & V23 & $\begin{array}{l}\text { Me gustar hacer cosas que impliquen sobresaltos } \\
\text { Me gusta experimentar cosas nuevas y excitantes, aunque } \\
\text { tenga que romper las normas } \\
\text { Prones }\end{array}$ \\
& V25 & $\begin{array}{c}\text { Prefiero a los amigos que son apasionados y } \\
\text { poco predecibles }\end{array}$ \\
\hline
\end{tabular}

Fuente: Elaboración propia.

A pesar de que los resultados de este modelo de medida indican que es estadísticamente significativo $\left[\chi^{2}(75)=210,709, p=0,000\right]$, es preciso señalar que este estadístico depende del tamaño de la muestra analizada, de ahí la necesidad de analizar otros indicadores de ajuste. A este respecto, los resultados obtenidos muestran como otros indicadores de ajuste global del modelo se encuentran dentro de los valores recomendados por la literatura (CFI=0,91, NFI=0,87, TLI=0,86, RMSEA=0,06), por lo que se puede concluir que el modelo especificado reproduce adecuadamente la matriz de covarianzas observada. Este modelo de medida muestra un ajuste satisfactorio, ya que el valor de CFI se sitúa entre 0,90 y 0,95 y el valor de RMSEA no supera el máximo recomendado de 0,08 (Mathieu y Taylor, 2006). Siguiendo a Anderson y Gerbing (1988) y como se observa en la Tabla 6, el modelo muestra una fiabilidad individual aceptable, ya que la relación existente entre cada ítem y su respectiva dimensión es estadísticamente significativa con pesos de regresión estandarizados próximos o superiores en su mayoría a 0,7 y con valores del estadístico t también significativos. En cuanto a las medidas de consistencia interna de cada una de las dimensiones, los valores del indicador de fiabilidad compuesta (FC) alcanzan valores muy cercanos a 0,70 y los de la varianza extraída (AVE) a 0,50. Los valores del alfa de Cronbach corroboran los obtenidos en la fiabilidad compuesta. Estos resultados indican que el modelo de medida es fiable.

Tabla 6

ANÁLISIS FACTORIAL CONFIRMATORIO DE LA ESCALA DE PERSONALIDAD*

\begin{tabular}{ccccc}
\hline Relaciones causales & $\begin{array}{c}\text { Estimadores } \\
\text { estandarizados }\end{array}$ & $\boldsymbol{t}$ & $\boldsymbol{p}$ & $\begin{array}{c}\text { Consistencia } \\
\text { interna }\end{array}$ \\
\hline $\mathrm{V} 7 \quad \leftarrow$ Extroversión & 0,716 & & & $\mathrm{FC}=0,688$ \\
$\mathrm{~V} 8 \mathrm{r} \quad \leftarrow$ Extroversión & 0,553 & 9,187 & 0,000 & $\begin{array}{l}\mathrm{AVE}=0,427 \\
\mathrm{a}=0,678\end{array}$ \\
$\mathrm{~V} 9 \quad \leftarrow$ Extroversión & 0,679 & 10,364 & 0,000 & \\
\hline
\end{tabular}




\begin{tabular}{|c|c|c|c|c|c|}
\hline \multicolumn{2}{|r|}{ Relaciones causales } & \multirow{2}{*}{$\begin{array}{c}\begin{array}{c}\text { Estimadores } \\
\text { estandarizados }\end{array} \\
0,744\end{array}$} & \multirow[t]{2}{*}{$t$} & \multirow[t]{2}{*}{$p$} & \multirow{3}{*}{$\begin{array}{l}\begin{array}{c}\text { Consistencia } \\
\text { interna }\end{array} \\
\mathrm{FC}=0,739 \\
\mathrm{AVE}=0,586 \\
\alpha=0,738\end{array}$} \\
\hline V10 & $\leftarrow$ Agradabilidad & & & & \\
\hline V11 & $\leftarrow$ Agradabilidad & 0,787 & 10,371 & 0,000 & \\
\hline V13 & $\leftarrow$ Responsabilidad & 0,532 & & & \multirow{3}{*}{$\begin{array}{l}\mathrm{FC}=0,622 \\
\mathrm{AVE}=0,357 \\
\alpha=0,609\end{array}$} \\
\hline V14r & $\leftarrow$ Responsabilidad & 0,688 & 7,161 & 0,000 & \\
\hline V15r & $\leftarrow$ Responsabilidad & 0,562 & 7,101 & 0,000 & \\
\hline V17 & $\leftarrow$ Neurotismo & 0,611 & & & \multirow{2}{*}{$\begin{array}{l}\mathrm{FC}=0,599 \\
\mathrm{AVE}=0,428 \\
\alpha=0,594\end{array}$} \\
\hline V18 & $\leftarrow$ Neurotismo & 0,695 & 4,465 & 0,000 & \\
\hline V19 & $\leftarrow \begin{array}{l}\text { Apertura a la } \\
\text { experiencia }\end{array}$ & 0,732 & & & \multirow{2}{*}{$\begin{array}{l}\mathrm{FC}=0,616 \\
\mathrm{AVE}=0,447 \\
\alpha=0,605\end{array}$} \\
\hline V20 & $\leftarrow \begin{array}{l}\text { Apertura a la } \\
\text { experiencia }\end{array}$ & 0,599 & 10,072 & 0,000 & \\
\hline V23 & $\leftarrow \begin{array}{l}\text { Búsqueda de } \\
\text { sensaciones }\end{array}$ & 0,699 & & & \multirow{3}{*}{$\begin{array}{l}\mathrm{FC}=0,758 \\
\mathrm{AVE}=0,512 \\
\alpha=0,750\end{array}$} \\
\hline V24 & $\leftarrow \begin{array}{l}\text { Búsqueda de } \\
\text { sensaciones }\end{array}$ & 0,792 & 12,271 & 0,000 & \\
\hline V25 & $\leftarrow \begin{array}{l}\text { Búsqueda de } \\
\text { sensaciones }\end{array}$ & 0,649 & 11,263 & 0,000 & \\
\hline
\end{tabular}

Fuente: Elaboración propia.

* Los ítems V8, V14 y V15 han sido recodificados en sus versiones opuestas en los AFC para que todos los ítems tuvieran el mismo sentido, reflejándose al añadir "r" al final del código de la variable (V8r, V14r y V15r).

\section{Contraste de hipótesis}

Como paso previo a la contrastación de las hipótesis formuladas, en la Tabla 7 se recogen las medias y las desviaciones típicas de las seis dimensiones de la personalidad, que indican que los turistas de ocio que visitan Gran Canaria se consideran a sí mismos principalmente como agradables $(M=5,93)$, responsables $(M=5,33)$, abiertos a nuevas experiencias $(M=5,14)$ y extrovertidos $(M=4,74)$. 
Tabla 7

ANÁLISIS DESCRIPTIVO DE LAS DIMENSIONES DE LA PERSONALIDAD

\begin{tabular}{lcc}
\hline \multicolumn{1}{c}{ DIMENSIONES } & MEDIA & D.T. \\
\hline Extroversión & 4,74 & 1,19 \\
Agradabilidad & 5,93 & 0,94 \\
Responsabilidad & 5,33 & 1,19 \\
Neurotismo & 3,12 & 1,43 \\
Apertura a la experiencia & 5,14 & 1,13 \\
Búsqueda de sensaciones & 4,03 & 1,46 \\
\hline
\end{tabular}

Fuente: Elaboración propia.

Para conocer la idoneidad de la personalidad del turista como criterio de segmentación es necesario conocer la influencia de las características sociodemográficas del turista en la personalidad, dando respuesta, por tanto, a las hipótesis planteadas. Para ello se ha realizado un test de diferencia de medias, utilizando el test de la $t$ para muestras independientes o el análisis ANOVA de un factor, según proceda. Más concretamente y atendiendo a la revisión de la literatura, se ha procedido a analizar la influencia del género, la edad, el nivel de estudios, la clase social y la nacionalidad en el nivel de los rasgos de personalidad.

Los resultados que se recogen en la Tabla 8 permiten afirmar que el género es una variable sociodemográfica que influye en el nivel de neurotismo $(t=3,207, p=0,001)$ y en el de búsqueda de sensaciones $(t=2,069, p=0,039)$, siendo las mujeres las que presentan un mayor grado de neurotismo $(\mathrm{M}=2,90$ y $\mathrm{M}=3,33$ para hombres y mujeres, respectivamente) y los hombres un mayor grado de búsqueda de sensaciones ( $\mathrm{M}=4,17$ y $\mathrm{M}=3,88$ para hombres y mujeres, respectivamente). Por tanto, y dado que el género influye en dos de los seis rasgos de personalidad podemos aceptar la hipótesis H1.

Tabla 8

INFLUENCIA DEL GÉNERO DEL TURISTA EN SUS RASGOS DE PERSONALIDAD

\begin{tabular}{lccc}
\hline \multicolumn{1}{c}{ DIMENSIONES } & HOMBRES & MUJERES & $\boldsymbol{t}$ \\
& & 4,72 & 0,295 \\
Extroversión & 4,76 & $(1,4)$ & $(0,768)$ \\
& $(1,25)$ & 5,98 & 1,192 \\
Agradabilidad & 5,87 & $(0,85)$ & $(0,234)$ \\
& $(1,02)$ & 5,30 & 0,440 \\
Responsabilidad & 5,35 & $(1,17)$ & $(0,660)$ \\
\hline
\end{tabular}




\begin{tabular}{lccc}
\hline \multicolumn{1}{c}{ DIMENSIONES } & HOMBRES & MUJERES & $\begin{array}{c}\boldsymbol{t} \\
\boldsymbol{p} \boldsymbol{)}\end{array}$ \\
\hline \multirow{2}{*}{ Neurotismo } & 2,90 & 3,30 & 3,207 \\
& $(1,40)$ & $(1,43)$ & $(0,001)$ \\
Apertura a la experiencia & 5,22 & 5,07 & 1,342 \\
& $(1,16)$ & $(1,10)$ & $(0,180)$ \\
Búsqueda de sensaciones & 4,17 & 3,88 & 2,069 \\
& $(1,45)$ & $(1,47)$ & $(0,039)$ \\
\hline
\end{tabular}

Fuente: Elaboración propia.

Como se desprende de los resultados del análisis ANOVA realizado (véase Tabla 9), la edad del turista influye en los niveles de tres de los seis rasgos de personalidad: responsabilidad $(F=3,395, p=0,005)$, neurotismo $(F=2,113, p=0,063)$ y búsqueda de sensaciones $(F=12,476, p=0,000)$. En términos generales, puede decirse que el nivel de responsabilidad aumenta a medida que se incrementa la edad, aunque a partir de los 64 años vuelve a bajar de forma significativa. En cuanto al neurotismo, los datos indican que este rasgo es más acusado entre la población más joven y las más adulta. Finalmente, como era de esperar, la búsqueda de sensaciones disminuye de forma considerable a medida que aumenta la edad ( $M=4,56$ y $M=2,99$ para turistas entre 16-24 años y más de 64 años, respectivamente). Por tanto, y dado que la edad influye en tres de los seis rasgos de personalidad es posible aceptar la hipótesis $\mathrm{H} 2$.

Tabla 9

INFLUENCIA DE LA EDAD DEL TURISTA EN SUS RASGOS DE PERSONALIDAD

\begin{tabular}{|c|c|c|c|c|c|c|c|}
\hline DIMENSIONES & $\begin{array}{c}\mathbf{1 6 - 2 4} \\
\text { años }\end{array}$ & $\begin{array}{c}25-34 \\
\text { años }\end{array}$ & $\begin{array}{c}35-44 \\
\text { años }\end{array}$ & $\begin{array}{c}45-54 \\
\text { años }\end{array}$ & $\begin{array}{c}\text { 55-64 } \\
\text { años }\end{array}$ & $\begin{array}{l}\text { Más de } \\
64 \text { años }\end{array}$ & $\begin{array}{c}F \\
(p)\end{array}$ \\
\hline Extroversión & $\begin{array}{c}4,80 \\
(1,09)\end{array}$ & $\begin{array}{c}4,84 \\
(1,14)\end{array}$ & $\begin{array}{c}4,64 \\
(1,33)\end{array}$ & $\begin{array}{c}4,61 \\
(1,13)\end{array}$ & $\begin{array}{c}4,58 \\
(1,27)\end{array}$ & $\begin{array}{c}4,62 \\
(1,23)\end{array}$ & $\begin{array}{c}0,652 \\
(0,660)\end{array}$ \\
\hline Agradabilidad & $\begin{array}{c}5,85 \\
(0,88)\end{array}$ & $\begin{array}{c}5,96 \\
(1,04)\end{array}$ & $\begin{array}{c}5,86 \\
(0,94)\end{array}$ & $\begin{array}{c}5,92 \\
(0,82)\end{array}$ & $\begin{array}{c}5,95 \\
(0,73)\end{array}$ & $\begin{array}{c}6,13 \\
(0,69)\end{array}$ & $\begin{array}{c}0,456 \\
(0,809)\end{array}$ \\
\hline Responsabilidad & $\begin{array}{c}5,06 \\
(1,17)\end{array}$ & $\begin{array}{c}5,18 \\
(1,21)\end{array}$ & $\begin{array}{c}5,52 \\
(1,18)\end{array}$ & $\begin{array}{c}5,44 \\
(1,15)\end{array}$ & $\begin{array}{c}6,05 \\
(0,88)\end{array}$ & $\begin{array}{c}5,49 \\
(1,10)\end{array}$ & $\begin{array}{c}3,395 \\
(0,005)\end{array}$ \\
\hline Neurotismo & $\begin{array}{c}3,03 \\
(1,44)\end{array}$ & $\begin{array}{c}3,34 \\
(1,44)\end{array}$ & $\begin{array}{c}2,87 \\
(1,31)\end{array}$ & $\begin{array}{c}2,82 \\
(1,34)\end{array}$ & $\begin{array}{c}3,15 \\
(1,58)\end{array}$ & $\begin{array}{c}3,27 \\
(1,68)\end{array}$ & $\begin{array}{c}2,113 \\
(0,063)\end{array}$ \\
\hline $\begin{array}{l}\text { Apertura a la } \\
\text { experiencia }\end{array}$ & $\begin{array}{c}5,28 \\
(0,85)\end{array}$ & $\begin{array}{c}5,17 \\
(1,14)\end{array}$ & $\begin{array}{c}5,05 \\
(1,21)\end{array}$ & $\begin{array}{c}4,90 \\
(1,25)\end{array}$ & $\begin{array}{c}5,48 \\
(1,12)\end{array}$ & $\begin{array}{c}5,19 \\
(1,00)\end{array}$ & $\begin{array}{c}1,111 \\
(0,354)\end{array}$ \\
\hline $\begin{array}{l}\text { Búsqueda de } \\
\text { sensaciones }\end{array}$ & $\begin{array}{c}4,56 \\
(1,36)\end{array}$ & $\begin{array}{c}4,41 \\
(1,36)\end{array}$ & $\begin{array}{c}3,76 \\
(1,30)\end{array}$ & $\begin{array}{c}3,20 \\
(1,46)\end{array}$ & $\begin{array}{c}3,48 \\
(1,58)\end{array}$ & $\begin{array}{c}2,99 \\
(1,51)\end{array}$ & $\begin{array}{l}12,476 \\
(0,000)\end{array}$ \\
\hline
\end{tabular}

Fuente: Elaboración propia. 
Antes de proceder a analizar la influencia del nivel de estudios en los niveles de los rasgos de personalidad, se consideró oportuno agrupar las categorías "Sin estudios" y "Primaria o ESO" dado el bajo nivel muestral de la categoría "Sin estudios", ya que ello afectaría al nivel de fiabilidad de los resultados. Como se desprende de los resultados del análisis ANOVA, recogidos en la Tabla 10, el nivel de estudios sólo influye en el nivel de la apertura a la experiencia, aunque con un nivel de significación del 6,5\%, donde los turistas con menor nivel de formación muestran un menor valor en este rasgo de personalidad $(\mathrm{M}=4,93)$. Por tanto, y dado que el nivel de estudios sólo influye en uno de los seis rasgos de personalidad, pero con un nivel de significación superior al $5 \%$, se ha decidido rechazar la hipótesis $\mathrm{H} 3$.

Tabla 10

INFLUENCIA DEL NIVEL DE ESTUDIOS DEL TURISTA EN SUS RASGOS DE PERSONALIDAD

\begin{tabular}{lccccc}
\hline DIMENSIONES & $\begin{array}{c}\text { Sin estudios/ } \\
\text { Primaria o ESO } \\
\text { (ESO, FP1, } \\
\text { EGB) }\end{array}$ & $\begin{array}{c}\text { Bachiller } \\
\text { o FP2 }\end{array}$ & $\begin{array}{c}\text { Universitarios } \\
\text { medios }\end{array}$ & $\begin{array}{c}\text { Universitarios } \\
\text { superiores }\end{array}$ & $\begin{array}{c}\boldsymbol{F} \\
(\boldsymbol{p})\end{array}$ \\
\hline Extroversión & 4,57 & 4,77 & 4,92 & 4,70 & 1,403 \\
& $(1,11)$ & $(1,22)$ & $(1,16)$ & $(1,22)$ & $(0,241)$ \\
Agradabilidad & 5,87 & 5,91 & 5,79 & 6,03 & 1,501 \\
& $(0,93)$ & $(1,03)$ & $(0,92)$ & $(0,91)$ & $(0,214)$ \\
Responsabilidad & 5,55 & 5,31 & 5,22 & 5,30 & 1,278 \\
& $(1,11)$ & $(1,24)$ & $(1,19)$ & $(1,19)$ & $(0,282)$ \\
Neurotismo & 3,07 & 3,14 & 3,04 & 3,17 & 0,241 \\
& $(1,42)$ & $(1,41)$ & $(1,29)$ & $(1,52)$ & $(0,868)$ \\
Apertura a la & & & & & \\
experiencia & 4,93 & 5,21 & 5,00 & 5,28 & 2,425 \\
Búsqueda de & $(1,26)$ & $(1,18)$ & $(1,00)$ & $(1,09)$ & $(0,065)$ \\
sensaciones & 3,80 & 4,18 & 3,88 & 4,13 & 1,618 \\
& $(1,35)$ & $(1,48)$ & $(1,52)$ & $(1,46)$ & $(0,184)$ \\
\hline
\end{tabular}

Fuente: Elaboración propia.

El reducido tamaño muestral de la clase social etiquetada como baja aconseja que sea recodificada con la categoría media-baja para garantizar la fiabilidad de los resultados del análisis ANOVA. Tal y como se desprende de los datos que se muestran en la Tabla 11, la clase social, al igual que el nivel de estudios, sólo afecta a la apertura a la experiencia $(F=4,628, p=0,003)$, apreciándose un incremento en el nivel de este rasgo de personalidad a medida que aumenta la clase social $(M=4,77$ y $M=5,32$ para turistas de clase social media-baja y baja y alta, respectivamente). Por tanto, y dado que la clase social influye 
al menos en uno de los seis rasgos de personalidad con un nivel de significación inferior al 5\%, se ha decidido aceptar la hipótesis H4.

Tabla 11

INFLUENCIA DE LA CLASE SOCIAL DEL TURISTA EN SUS RASGOS DE PERSONALIDAD

\begin{tabular}{lccccc}
\hline \multicolumn{1}{c}{ DIMENSIONES } & Alta & Media-Alta & Media-Media & $\begin{array}{c}\text { Media-Baja/ } \\
\text { Baja }\end{array}$ & $\begin{array}{c}\boldsymbol{F} \\
(\boldsymbol{p})\end{array}$ \\
\hline Extroversión & 5,05 & 4,75 & 4,77 & 4,52 & 1,908 \\
& $(1,19)$ & $(1,19)$ & $(1,18)$ & $(1,22)$ & $(0,128)$ \\
Agradabilidad & 6,00 & 5,87 & 5,98 & 5,84 & 0,609 \\
& $(0,77)$ & $(0,96)$ & $(0,98)$ & $(0,96)$ & $(0,610)$ \\
Responsabilidad & 5,37 & 5,20 & 5,44 & 5,35 & 1,096 \\
& $(1,13)$ & $(1,20)$ & $(1,21)$ & $(1,19)$ & $(0,350)$ \\
Neurotismo & 3,20 & 3,04 & 3,19 & 3,13 & 0,342 \\
& $(1,36)$ & $(1,51)$ & $(1,41)$ & $(1,34)$ & $(0,795)$ \\
Apertura a la & 5,32 & 5,30 & 5,11 & 4,77 & 4,628 \\
experiencia & $(1,15)$ & $(0,99)$ & $(1,14)$ & $(1,30)$ & $(0,003)$ \\
Búsqueda de & 4,23 & 4,02 & 4,07 & 3,74 & 1,413 \\
sensaciones & $(1,34)$ & $(1,47)$ & $(1,41)$ & $(1,56)$ & $(0,238)$ \\
\hline
\end{tabular}

Fuente: Elaboración propia.

Finalmente, los resultados, que se recogen en la Tabla 12 indican que la nacionalidad influye en todos los rasgos de personalidad a excepción de la agradabilidad $(F=0,269$, $p=0,98)$. Los resultados indican que: 1$)$ los españoles son los más extrovertidos $(M=5,259)$, situándose el resto de nacionalidades en niveles muy similares que oscilan entre 4,60 y $4,80 ; 2)$ los alemanes son los más responsables $(M=5,71)$, seguidos a cierta distancia de los holandeses $(M=5,31)$ y el resto con niveles entre 4,99 y 5,$19 ; 3)$ los españoles son los más neuróticos $(M=3,60)$, situándose el resto a cierta distancia con niveles que se sitúan entre 2,48 y 3,$21 ; 4)$ los españoles son los más proclives a nuevas experiencias $(\mathrm{M}=5,60)$, posicionándose el resto a cierta distancia con niveles que oscilan entre 4,99 y 5,14; y, finalmente, 5) los turistas españoles y los del Reino Unido e Irlanda del Norte son los que puntúan más alto en la búsqueda de sensaciones ( $M=4,55$ y $M=4,20$, respectivamente), encontrándose las demás nacionalidades en niveles inferiores a 4. Por tanto, puede afirmarse que se acepta la hipótesis H5. 
Tabla 12

INFLUENCIA DE LA NACIONALIDAD DEL TURISTA EN SUS RASGOS DE PERSONALIDAD

\begin{tabular}{lcccccc}
\hline DIMENSIONES & Alemania & $\begin{array}{c}\text { Reino Unido } \\
\text { e Irlanda del } \\
\text { Norte }\end{array}$ & $\begin{array}{c}\text { España } \\
\text { (Península y } \\
\text { Baleares) }\end{array}$ & $\begin{array}{c}\text { Países } \\
\text { Nórdicos }\end{array}$ & Holanda & $\begin{array}{c}\boldsymbol{F} \\
(\boldsymbol{p})\end{array}$ \\
\hline Extroversión & 4,60 & 4,73 & 5,29 & 4,60 & 4,80 & 3,598 \\
& $(1,09)$ & $(1,25)$ & $(1,18)$ & $(1,22)$ & $(1,08)$ & $(0,007)$ \\
Agradabilidad & 5,88 & 5,94 & 6,03 & 5,93 & 6,01 & 0,269 \\
& $(0,89)$ & $(0,92)$ & $(0,99)$ & $(1,08)$ & $(0,80)$ & $(0,898)$ \\
Responsabilidad & 5,71 & 4,99 & 5,10 & 5,19 & 5,31 & 6,013 \\
& $(1,17)$ & $(1,22)$ & $(1,07)$ & $(1,17)$ & $(1,13)$ & $(0,000)$ \\
Neurotismo & 3,14 & 3,21 & 3,60 & 2,86 & 2,78 & 2,979 \\
Apertura a la & $(1,42)$ & $(1,48)$ & $(1,47)$ & $(1,25)$ & $(1,44)$ & $(0,019)$ \\
experiencia & 5,14 & 4,99 & 5,60 & 5,01 & 5,07 & 2,798 \\
Búsqueda de & $(1,25)$ & $(1,10)$ & $(1,15)$ & $(1,09)$ & $(0,82)$ & $(0,026)$ \\
sensaciones & 3,80 & 4,20 & 4,55 & 3,69 & 3,93 & 4,013 \\
\hline
\end{tabular}

Fuente: Elaboración propia.

\section{CONCLUSIONES, IMPLICACIONES PRÁCTICAS Y LIMITACIONES}

A lo largo de la amplia literatura analizada para la realización de este trabajo, se ha podido comprobar la importancia de la personalidad del turista en la elección de un destino. Sin embargo, no se han encontrado trabajos que relacionen los rasgos de personalidad del turista de sol y playa con sus características sociodemográficas, de ahí la relevancia de este estudio, tanto en la vertiente académica como profesional.

\section{Aportaciones académicas}

Como principal aportación académica, destaca la validación del modelo de medida de la personalidad en el que se hace una consideración conjunta de los cinco grandes rasgos de personalidad y la búsqueda de sensaciones. En el diseño de la escala de la personalidad se ha partido de la escala TIPI, reforzada con cinco ítems de la escala BFI, para medir los Cinco Grandes, e incorporando la escala de búsqueda de sensaciones BSSS de Hoyle (2002).

Si bien, podemos destacar algunos trabajos que avalan la influencia de las características sociodemográficas analizadas en la personalidad (Burton et al., 2007; Laesser y Zehrer, 2012; Tan y Tang, 2013: Pizam et al., 2004; Xu et al., 2012; Zuckerman, 2004), es preciso señalar que adolecen del carácter integrador de esta investigación y de la utilización conjunta de los cinco grandes rasgos de personalidad y la búsqueda de sensaciones, de ahí la relevancia académica de este trabajo. 
A modo de síntesis, las principales conclusiones de la investigación empírica son las siguientes y que pueden ser de apoyo para futuros trabajos que se planteen bajo esta misma óptica: 1) el género es una variable sociodemográfica que influye en el nivel de neurotismo y en el de búsqueda de sensaciones; 2) la edad influye en los niveles de tres de los seis rasgos de personalidad: responsabilidad, neurotismo y búsqueda de sensaciones; 3 ) el nivel de estudios sólo influye en el nivel de la apertura a la experiencia, aunque con un nivel de significación del $6,5 \%$; 4) la clase social sólo afecta a la apertura a la experiencia, apreciándose un incremento en el nivel de este rasgo de personalidad a medida que aumenta la clase social; y 5) la nacionalidad influye en todos los rasgos de personalidad a excepción de la agradabilidad. Estos datos, en principio, confirman la influencia de las características sociodemográficas de los turistas de ocio. No obstante, es preciso señalar que, aunque las escasas evidencias empíricas recogidas en la literatura señalan que la escala de los cinco grandes rasgos de personalidad se caracteriza por la estabilidad e invariabilidad en función de las características sociodemográficas, a excepción del género, en este trabajo se aporta evidencias empíricas que sugieren la influencia de otras variables sociodemográficas en la personalidad.

\section{Contribuciones profesionales}

Con relación a las implicaciones prácticas de este estudio, los responsables de los destinos turísticos y las empresas turísticas que operan en los mismos podrían plantearse la idoneidad de considerar los rasgos de personalidad como criterio base para segmentar los mercados, además de las características sociodemográficas, que se corresponden con los criterios de segmentación más aplicados en el sector. A modo de ejemplo, turistas con un mismo género o edad podrían exhibir niveles diferenciados en los rasgos de personalidad. Asimismo, dado que en esta investigación se ha evidenciado que la nacionalidad influye en todos los rasgos de personalidad, a excepción de la agradabilidad, no sería aconsejable agrupar los mercados emisores considerando únicamente el idioma, y que ello implique agrupar turistas de varias nacionalidades (alemana, austriaca, suiza germano-parlante). Una de las razones por las que la personalidad no se utiliza en la segmentación del mercado es que no cumple con el requisito mensurabilidad (fácil de medir). La validación de la escala de medida utilizada en esta investigación para evaluar los rasgos de personalidad, compuesta por un reducido número de ítems, salva el obstáculo antes citado y, por consiguiente, se recomienda, como otra contribución profesional, la inclusión de esta escala en los instrumentos de medición global que utilizan los organismos responsables de la elaboración de estadísticas turísticas. La generación de información periódica sobre esta variable podría tener un impacto en la sociedad al permitir evaluar la evolución del destino turístico atendiendo al perfil de los visitantes. A modo de ejemplo, el crecimiento de turistas con rasgos optimistas de personalidad (extroversión, agradabilidad, apertura a la experiencia, búsqueda de sensaciones) podría indicar un posible cambio en sentido positivo en la imagen del destino. Esta actuación profesional, - que se ajusta a las recomendaciones de la teoría de autocongruencia, según la cual los turistas buscan destinos que se ajusten a su personalidad- tendría un impacto sobre todos los agentes turísticos del destino. 


\section{Limitaciones}

A modo de conclusión, si bien en el presente ensayo hemos seguido criterios de rigurosidad científica, se ha detectado algunas limitaciones que formulamos como dos recomendaciones para investigaciones futuras. En primer lugar, y con la finalidad de contribuir a la consolidación del conocimiento actual, se recomienda la realización de investigaciones adicionales que tomen como punto de partida las escalas desarrolladas en aras de mejorar su validez y fiabilidad. Y, en segundo lugar, sería conveniente replicar la investigación en otros destinos y contextos turísticos distintos al turismo de sol y playa.

\section{REFERENCIAS BIBLIOGRÁFICAS}

ABBATE, C.S. y NUOVO, S.D. (2013) "Motivation and personality traits for choosing religious tourism. A research on the case of Medjugorje". Current Issues in Tourism, 16 (5), 501-506.

ALLPORT, G.W. (1940): “The psychologist's frame of reference”. Psychological Bulletin, $37,1-28$.

ALLPORT, G.W. (1961): Patterns and growth in personality. New York: Holt, Rinehart \& Winston.

ANDERSON, J.C. y GERBING, D.W. (1988): "Structural equation modeling in practice: A review and recommended two-step approach". Psychological Bulletin, 103 (3), 411-423.

ARIFFIN, A.A.M., AHMAD, A.H. y ISHAK, N.K. (2008): “Corporate Meeting Destination Choice: The Influences of Consumption Value, Organizational Structure and Personality". International Journal of Hospitality \& Tourism Administration, 9 (4), 313-326.

BARRICK, M.R. y MOUNT, M.K. (1991): “The big five personality dimensions and job performance: A metaanalysis”. Personnel Psychology, 44 (1), 1-26.

BENET-MARTÍNEZ, V y JOHN, O.P. (1998): “Los Cinco Grandes across cultures and ethnic groups: Multitrait-multimethod analyses of the Big Five in Spanish and English". Journal of Personality and Social Psychology, 75 (3), 729-750.

BOKSBERGER, P., DOLNICAR S., LAESSER, C. y MELANIE RANDLE, M. (2011): "Self-Congruity Theory: To What Extent Does It Hold in Tourism?" Journal of Travel Research, 50 (4), 454-464.

BURNIK, S., JUG, S. y KAJTNA, T. (2008): "Sensation Seeking in slovenian female and male mountain climbers". Acta Universitatis Palackianae Olomucensis. Gymnica, 38 (3), 15-19.

BURTON, L., HAFETZ, J., y HENNINGER, D. (2007): “Gender differences in relational and physical aggression". Social Behavior and Personality, 35, 41-50.

CARVER, C.S. y SCHEIER, M.F. (2008): Perspectives on personality. Boston: Pearson/ Allyn and Bacon.

CATTELL, R.B. (1992): "Human motivation objectively, experimentally analysed". British Journal of Medical Psychology, 65, 237-243. 
CATTELL, R.B. y KRUG, S.E. (1986): "The number of factors in the 16PF: A review of the evidence with spezial emphasis on methodological problems". Educational and Psychological Measurement, 46 (3), 509-522.

CHANDLER, J.A. y COSTELLO, C.A. (2002): “A Profile of Visitors at Heritage Tourism Destinations in East Tennessee according to Plog's Lifestyle and Activity Level Preferences Model". Journal of Travel Research, 41, 161-166.

CLONINGER, S.C. (2003). Teorías de la personalidad. México: Pearson Education.

COHEN E. (1972): “Towards a sociology of international tourism”. Social Research, 39, 164-182.

COSTA, J.P., TERRACCIANO, A. y MCCRAE, R.R. (2001): “Gender differences in personality traits across cultures: Robust and surprising findings”. Journal of Personality and Social Psychology, 81 (2), 322-331.

COSTA, P.T Jr. y MCCRAE, R.R. (1988): "Personality in adulthood: A six year longitudinal study of self-reports and spouse rating on the NEO personality inventory". Journal of Personality and Social Psychology, 54 (5), 853-863.

COSTA, P.T. y MCCRAE, R.R. (1990): "Personality disorders and the five-factor model of personality". Journal of Personality Disorders, 4 (4), 362-371.

COSTA, P.T. y MCCRAE, R.R. (1992): "Four ways five factors are basic". Personality and Individual Differences, 13 (6), 653-665.

COSTA, P.T.Jr. y MCCRAE, R.R. (1989): The NEO-PI/FFI manal supplement. Odessa, FL: Psychological Assessment Resources.

CROMPTON, J.L. (1979): "Motivations for pleasure vacations". Annals of Tourism Research, 6 (4), 408-424.

DANN, G.M.S. (1977): “Anomie, ego-enhancement and tourism”. Annals of Tourism Research, 4 (4), 184-194.

EYSENCK, H.J. (1991): "Personality and Individual Differences. Dimensions of personality: 16, 5, or 3?-Criteria for a taxonomic paradigm”. Personality and Individual Differences, 12 (8), 773-790.

EYSENCK, M.W. y EYSENCK, H.J. (1978): “Impulsiveness and venturesomeness: their position in a dimensional system of personality description". Psychological Reports, 43, 1.247-1.255.

FELDT, T., METSÄPELTO, R.L., KINNUNEN, U. y PULKKINEN, L. (2007): “Sense of Coherence and Five-Factor Approach to Personality: Conceptual Relationships". European Psychologist, 12 (3), 165-172.

GOLDBERG, L.R. (1981): "Language and individual differences: The search for universals in personality lexicons". Review of Personality and Social Psychology, 2, 141-165.

GOLDBERG, L.R. (1990): "An alternative "description of personality": The Big-Five factor structure". Journal of Personality and Social Psychology, 59 (6), 1216-1229.

GOSLING, S.D., RENTFROW, P.J. y SWANN, W.B. (2003): "A very brief measure of the Big-Five personality domains". Journal of Research in Personality, 37, 504-528.

GRETZEL, U., MITSCHE, N., HWANG, Y.H. y FESENMAIER, D.R. (2004): “Tell me who you are and I will tell you where to go: use of travel personalities in destination recommendation systems". Information Technology \& Tourism, 7 (1), 3-12. 
HEE, O.C. y JOHARI, H. (2014): “A Conceptual Analysis of Personality Traits and Customer-Oriented Behaviour in the Health Tourism Hospitals". International Journal of Caring Sciences, 7 (2), 368-374.

HOLLAND, J.L. (1985): Making vocational choices: A theory of vocational personalities and work environments. New York: Prentice-Hall.

HOYLE, R.H., STEPHENSON, M.T., PALMGREEN, P., LORCH, E.P. y DONOHEW, R.L. (2002): "Reliability and validity of a brief measure of sensation seeking". Personality and Individual Differences, 32, 401-414.

JACK, S.J. y RONAN, K.R. (1998): "Sensation seeking among high- and low-risk sports participants". Personality and Individual Differences, 25 (6), 1.063-1.083.

JACKSON, D.N., PAUNONEN, S.V., FRABONI, M. y GOFFIN, R.G. (1996): “A fivefactor versus a six-factor model of personality structure". Personality and Individual Differences, 20, 33-45.

JANI, D. (2014): "Big five personality factors and travel curiosity: are they related?". Anatolia, 25 (3), 444-456.

JOHARI, H. y HEE, O.C. (2013): "Personality traits and customer-oriented behavior in the health tourism hospitals in Malaysia”. International Journal of Trade, Economics and Finance, 4 (4), 213-216.

JOHN, O. y SRIVASTAVA, S. (1999): The Big Five Trait Taxonomy: History, Measurement, and Theoretical Perspectives. University of California, Berkeley.

KALMUS, V., REALO, A. y SIIBAK, A. (2011): "Motives for Internet use and their relationships with personality traits and socio-demographic factors". Trames, 15 (4), 385-403.

KROESEN, M. y HANDY, S. (2014): “The influence of holiday-taking on affect and contentment". Annals of Tourism Research, 45, 89-101.

LAESSER, C. y ZEHRER, A. (2012): “Tell me who you think you are and I tell you how you travel: Exploring the viability of market segmentation by means of travelers' stated personality: Insights from a mature market (Switzerland)". Tourism Analysis, 17 (3), 285-298.

LARSEN, R.J. y BUSS, D.M. (2008): Personality Psychology. Texas: McGrau Hill.

LEDESMA, R.D., SÁNCHEZ, R. y DÍAZ-LÁZARO, C.M. (2011): “Adjective checklist to assess the big five personality factors in the Argentine population". Journal of Personality Assessment, 93 (1), 46-55.

LLEWELLYN, D.J. y SÁNCHEZ, X. (2008): "Individual differences and risk taking in rock climbing". Psychology of Sport and Exercise, 9 (4), 413-426.

MATHIEU, J.E. y TAYLOR, S.R. (2006): "Clarifying conditions and decision points for mediational type inferences in organizational behavior". Journal of Organizational Behavior, 27 (8), 1031-1056.

MCCRAE, R.R. y COSTA, P.T.Jr. (1987): "Validation of the Five-Factor Model of personality across instruments and observers". Journal of Personality and Social Psychology, 52 (1), 81-90.

MCCRAE, R.R. y COSTA, P.T. (2003). Personality in Adulthood: A Five-factor Theory Perspective. Nueva York: The Guilford Press. 
MCCRAE, R.R., COSTA, P.T., OSTENDORF, F., ANGLEITNER, A., HŘEBÍČKOVÁ, M., AVIA, M.D., SANZ, J., SÁNCHEZ-BERNARDOS, M.L., KUSDIL, M.E., WOODFIELD, R., SAUNDERS, P.R. y SMITH, P.B. (2000): "Nature over nurture: Temperament, personality, and life span development". Journal of Personality and Social Psychology, 78 (1), 173-186.

MUELLER, S. y PETERS, M. (2008): “The personality of freestyle snowboarders: Implications for product development". Tourism, 56 (4), 339-354.

PASSAFARO, P., CINI, F., BOI, L., D’ANGELO, M., HEERING, M.S., LUCHETTI, L., MANCINI, A., MARTEMUCCI, V., PACELlA, G., PATRIZI, F., SASSU, F. y TRIOLO, M. (2015): “The "sustainable tourist": Values, attitudes, and personality traits". Tourism and Hospitality Research, 0 (0), 1-15.

PAUNONEN, S.V. y JACKSON, D.N. (2000): "What is beyond the big five? Plenty!" Journal of Personality, 68, 821-835.

PIZAM, A. (2004): "The relationship between risk-taking, sensation-seeking, and the tourist behavior of young adults: A cross-cultural study". Journal of Travel Research, $42(3), 251-260$.

PIZAM, A., JEONG, G REICHEL, A., BOEMMEL, V.H., LUSSON, J.M., STEYNBERG, L., STATE-COSTACHE, O., VOLO, S., KROESBACHER, C., KUCEROVA, J. y MONTMANY, N. (2004): "The relationship between risk-taking, sensation-seeking, and the tourist behavior of young adults: A cross-cultural study". Journal of Travel Research, 251 (42), 251-260.

PIZAM, A., REICHEL, A. y URIELY, N. (2002): "Sensation seeking and tourist behaviour". Journal of Hospitality and Leisure Marketing, 9 (2), 17-33

PLOG, S.C. (1991): A carpenter's tools re-visited: Measuring allocentrism and psychocentrism properly.The first time". Journal of Travel Research, 29 (4), 51-52.

PLOG, S.C. (1995): Vacation Places Rated. Redondo Beach, CA: Fielding Worldwide.

SCHNEIDER y VOGT, (2012): “Applying the 3M model of personality and motivation to adventure travelers". Journal of Travel Research, 51 (6), 704-716.

SMITH, V.L. (1977): Anfitriones e invitados. Antropología del turismo. Madrid: Endymion.

TAN, W. y TANG, C. (2013). Does personality predict tourism information search and feedback behaviour? Current Issues in Tourism, 16 (4), 388-406.

TOK, S. (2011): "The big five personality traits and risky sport participation". Social Behavior and Personality, 39 (8), 1105-1112.

WAGNER, A.M. y HOULIHAN, D.D. (1994): "Sensation seeking and trait anxiety in hang-glider pilots and golfers". Personality and Individual Differences, 16 (6), 975-977.

XU, S., BARBIERI, C., STANIS, S.W. y MARKET, P.S. (2012): "Sensation-seeking attributes associated with storm-chasing tourists: Implications for future engagement". International Journal of Tourism Research, 14, 269-284.

ZUCKERMAN, M. y EYSENCK, H.J. (1978): "Sensation seeking in England and America: Cross-cultural, age, and sex comparisons". Journal of Consulting and Clinical Psychology, 46 (1), 139-149. 
ZUCKERMAN, M. (1979): “Attribution of success and failure revisited, or: The motivational bias is alive and well in attribution theory". Journal of Personality, 47 (2), 245-287.

ZUCKERMAN, M. (1983): A biological theory of sensation seeking. Hillsdale, NJ: M. Zuckerman (Ed.).

ZUCKERMAN, M. (1984): "Sensation seeking: a comparative approach to a human trait". Beahavioral and Brain Science, 7, 413-471.

ZUCKERMAN, M. (1990): “The psychophysiology of sensation seeking”. Journal of Personality, 58 (1), 313-345.

ZUCKERMAN, M. (1994): Behavioral expressions and biosocial bases of sensation seeking. New York: Cambridge University Press. 
